\title{
Parametric calculation of pulse transformer with open magnetic cores based on magnetostatic-field theory
}

\author{
Bin-xiong Yu and Jin-liang Liu \\ College of Opto-electronic Science and Engineering, National University of Defense Technology, \\ Changsha, Hunan, 410073, People's Republic of China \\ (Received 20 July 2012; published 18 January 2013)
}

\begin{abstract}
An analytical method for calculating the magnetostatic field of a pulse transformer with open magnetic cores is put forward in this paper, and formulas for calculating inductances of a small aspect-ratio transformer are derived. In comparison to results calculated by finite element magnetostatic-field simulations, the calculated values of inductance of primary winding $L_{1}$ and the inductance of secondary winding $L_{2}$ have a relative error of about 5\%, while the error of the coupling coefficient $(k)$ is less than $2 \%$. Meanwhile, the effect of current nonuniformity in the primary winding on magnetizing inductance is studied. According to the calculated results, this effect reduces the magnetizing inductance and the coupling coefficient of the transformer, and can lead to an overvoltage phenomenon on the secondary winding. A small aspect-ratio pulse transformer with open magnetic cores is developed, which has a small size of $250 \mathrm{~mm} \times 150 \mathrm{~mm}$ in length and diameter, respectively. Inductances of the transformer are measured. The measured results conform to the law obtained in this work. Tests of the pulsed transformer are carried out. Experimental results show that the transformer can export a high-voltage pulse with an amplitude of $310 \mathrm{kV}$ and full width at half maximum of $1 \mu \mathrm{s}$.
\end{abstract}

DOI: 10.1103/PhysRevSTAB.16.010401

PACS numbers: $84.70 .+\mathrm{p}, 84.30 . \mathrm{Ng}$

\section{INTRODUCTION}

Intense electron-beam accelerators (IEBA) based on the Blumlein line are widely used in a great variety of applications such as lasers, high power microwave generators, $\mathrm{x}$-pinch and x-ray generation, and so on. In the traditional IEBA, the Marx generator was usually used to charge the pulse forming line (PFL) of IEBA. However, it was difficult to operate under a high repetition rate. In order to improve the repetition rate of IEBA, tesla transformers with compact axial structure and high coupling coefficient were widely used to charge PFL. Mesyats and his partners put the tesla transformer with open magnetic cores into a PFL [1] for the first time, and developed "Sinus"-series accelerators successfully. Transformer oil was used as the dielectric material of the PFL with impedance of $50 \Omega$, and the repetition frequency of the Sinus-type accelerator was up to $100 \mathrm{~Hz}$. At the same time, people did much work on an accelerator based on a water PFL charged by a tesla transformer [2-7], which operated repetitively with low impedance. It was used to drive a low-impedance microwave source or generate electron beam for material surface treatment. The tesla transformer was a key part and foundation of this kind of accelerator [8-10]. Korovin extrapolated the parametric calculation formulas of the open-core type of tesla transformer with a large aspect ratio (large

Published by the American Physical Society under the terms of the Creative Commons Attribution 3.0 License. Further distribution of this work must maintain attribution to the author(s) and the published article's title, journal citation, and DOI. aspect ratio represents the ratio of length and radius of the magnetic core is larger than 4:1) [11], and analyzed an interturn voltage distribution of the secondary winding. The coupling coefficient was calculated based on an exact magnetostatic-field theory [12]. The formula derived by Korovin in Ref. [11] was simple and convenient for engineering design, and it was suitable for compact transformers whose magnetic cores were partly inside the PFL. However, it became not correct enough for transformers whose magnetic cores were not inside the PFL, especially when transformers had a small aspect ratio or the permeability of the magnetic cores was not large enough. The formula in Ref. [12] gave the solution in series, so it is difficult to be used in engineering design. Moreover, both of the two methods were based on the magnetostatic-field condition, and the effects of frequency on inductances were not taken into consideration.

According to magnetostatic-field theory, an approximate analytic solution describing the magnet field in a transformer with an open magnetic core is put forward, and formulas for inductance calculations of the transformer are extrapolated. Then a pulse transformer with an open magnetic core is designed and the main parameters are calculated by four different methods. The first three methods all assume complete magnetostatic conditions so that the current in the primary winding is uniform: (1) Finite elements magnetostatic-field simulations, (2) using the formulas derived in this paper, and (3) the formula derived by Korovin in Ref. [11]. The fourth method, which is developed in this paper, does not require magnetostatic conditions and it allows for nonuniformity in the primary 


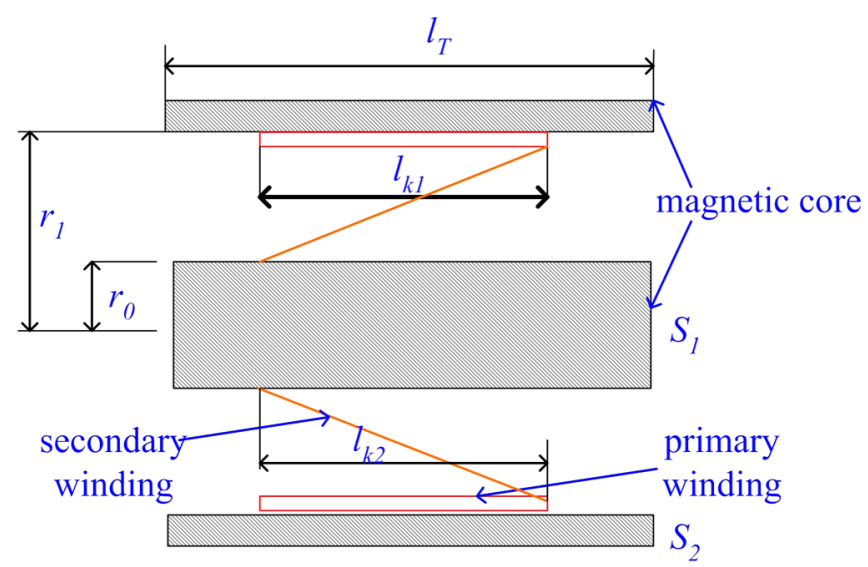

FIG. 1. Structure of the transformer with an open magnetic core.

winding as the frequency increases. These calculations all are compared with experimental inductance measurements in Sec. III.

\section{THEORETICAL ANALYSIS OF INDUCTANCES OF TRANSFORMER WINDINGS}

\section{A. Magnetostatic-field analysis}

The structure of a pulse transformer with an open magnetic core is shown in Fig. 1, where $r_{0}$ and $r_{1}$ represent the outer diameter of the inner magnetic core and the inner diameter of the outer magnetic core, and $l_{k 1}, l_{k 2}$ stand for the lengths of the primary winding and secondary winding, respectively. $l_{T}$ is the length of the magnetic core, whose relative permeability is $\mu_{r} . S_{1}$ and $S_{2}$ stand for effective cross-section areas of the inner and the outer magnetic cores. $N_{1}$ and $N_{2}$ represent the turn number of primary winding and secondary winding, respectively. Magnetic field distribution is shown in Fig. 2. Assuming that the radial magnetic field in the magnetic cores is uniform, the magnetic field of the inner and outer magnetic cores can be described by $H_{m 1}(z)$ and $H_{m 2}(z)$. The field in the spacing between the magnetic cores includes an axial and a radial component, which are $H_{g z}(z, r)$ and $H_{g r}(z, r)$, respectively. As the main magnetic flux of the transformer is mainly determined by $H_{m}, H_{g z}$ is ignored in the analysis at first.

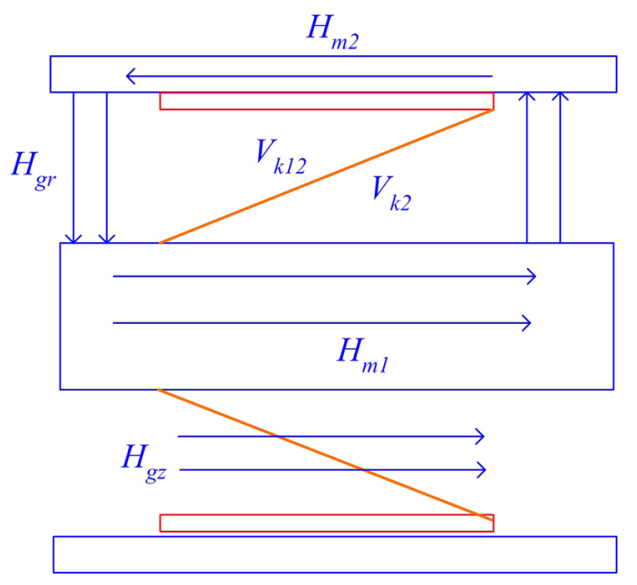

FIG. 2. Magnetic field distribution of the transformer.

Assuming the primary current defined as $I_{1}$ is uniformly distributed in primary winding which is located at the middle of magnetic core, and boundary conditions are the same at both ends of the magnetic core. Thus, the transformer has a symmetrical magnetic field distribution.

According to flux conservation,

$$
\mu_{r} \frac{\partial H_{m 1} S_{1}}{\partial z}+2 \pi r H_{g r}=0
$$

and

$$
H_{m 1} S_{1}=H_{m 2} S_{2} \text {. }
$$

From Ampere's circuital law,

$$
H_{m 1}+H_{m 2}+\int_{r_{0}}^{r_{1}} \frac{\partial H_{g r}}{\partial z} d r= \begin{cases}N_{1} I_{1} / l_{k 1} & 0<z<l_{k 1} / 2 \\ 0 & l_{k 1} / 2<z<l_{T} / 2 .\end{cases}
$$

Combining Eqs. (1)-(3), it is obtained that

$$
\begin{aligned}
(1 & \left.+S_{1} / S_{2}\right) H_{m 1}-\frac{\mu_{r} S_{1} \ln \left(r_{1} / r_{0}\right)}{2 \pi} \frac{\partial^{2} H_{m 1}}{\partial z^{2}} \\
& = \begin{cases}N_{1} I_{1} / l_{k 1} & 0<z<l_{k 1} / 2 \\
0 & l_{k 1} / 2<z<l_{T} / 2 .\end{cases}
\end{aligned}
$$

Then

$$
H_{m 1}(z)= \begin{cases}A \exp (-\omega z)+B \exp (\omega z)+S_{2} N_{1} I_{1} /\left(S_{1}+S_{2}\right) l_{k 1} & 0<z<l_{k 1} / 2 \\ A_{1} \exp (-\omega z)+B_{1} \exp (\omega z) & l_{k 1} / 2<z<l_{T} / 2\end{cases}
$$

where

$$
\omega=\sqrt{\frac{\left(1+S_{1} / S_{2}\right) 2 \pi}{\mu_{r} S_{1} \ln \left(r_{1} / r_{0}\right)}}
$$

The boundary conditions of Eq. (5) are

$$
H_{m 1}^{\prime}(0)=0, \quad H_{m 1}\left(l_{k 1}^{+} / 2\right)=H_{m 1}\left(l_{k 1}^{-} / 2\right), \quad H_{m 1}^{\prime}\left(l_{k 1}^{+} / 2\right)=H_{m 1}^{\prime}\left(l_{k 1}^{-} / 2\right) .
$$


However, there are four variables in Eq. (5), so one more boundary condition is required. According to the practical condition, we can get the last one boundary condition. Here, two familiar cases are taken into account.

Case 1.-The magnetic cores are inside metal of the PFL such as the pulse transformer in compact generator "SINUS120", and $H_{z}$ is close to zero at the ends of the magnetic core under high frequency because of the shield of metal.

That is,

$$
H_{m 1}\left(l_{T} / 2\right)=0
$$

Then, we get

$$
\begin{aligned}
H_{m 1}(z)= & -\frac{N_{1} I_{1} S_{2}}{\left(S_{1}+S_{2}\right) l_{k 1}} \frac{\cosh \left[\omega\left(l_{T}-l_{k 1}\right) / 2\right] \cosh (\omega z)}{\cosh \left(\omega l_{T} / 2\right)} \\
& +\frac{N_{1} I_{1} S_{2}}{\left(S_{1}+S_{2}\right) l_{k 1}}, \quad 0<z<\frac{l_{k 1}}{2} .
\end{aligned}
$$

Case 2.- Sometimes, we just need a discrete transformer, and the magnetic cores of the transformer are not inside metal. Magnetic cores are totally open at their ends. In this case, $H_{z}$ at the ends of the magnetic core is no more 0 . However, we can evaluate the magnet field in the area at $z>l_{T} / 2$. Assuming magnetic fields in the corresponding air are $H_{m 1^{\prime}}$ and $H_{g r}$, which correspond to $H_{m 1}$ and $H_{g r}$, and they go to 0 at infinity. So the solution of $H_{m 1^{\prime}}$ has a similar form with Eq. (5). That is,

$$
H_{m 1^{\prime}}=A_{2} \exp \left(-\omega_{1} z\right) \text {. }
$$

Here, $\omega_{1}$ is derived by substituting $\mu_{r}$ with 1 in Eq. (6). Using continuous conditions at $l_{T} / 2$, we get another restriction:

$$
A_{1}=B_{1} \frac{1+\sqrt{\mu_{r}}}{1-\sqrt{\mu_{r}}} \exp \left(\omega l_{T}\right)
$$

Then

$$
\begin{aligned}
H_{m 1}(z)= & -\frac{N_{1} I_{1} S_{2}}{\left(S_{1}+S_{2}\right) l_{k 1}} \frac{2\left\{\sqrt{\mu_{r}} \cosh \left[\omega\left(l_{T}-l_{k 1}\right) / 2\right]+\sinh \left[\omega\left(l_{T}-l_{k 1}\right) / 2\right]\right\}}{\sqrt{\mu_{r}} \cosh \left(\omega l_{T} / 2\right)+\sinh \left(\omega l_{T} / 2\right)} \cosh (\omega z) \\
& +\frac{N_{1} I_{1} S_{2}}{\left(S_{1}+S_{2}\right) l_{k 1}}, \quad 0<z<l_{k 1} / 2
\end{aligned}
$$

\section{B. Parametric calculation}

Only the axial component of the magnetic field $H_{z r}$ is needed when we calculate inductances of the transformer, and $H_{z r}$ is derived by

$$
\frac{\partial H_{g r}}{\partial z}-\frac{\partial H_{g z}}{\partial r}=0
$$

Namely,

$$
H_{g z}=\int_{r_{0}}^{r} \frac{\partial H_{g r}}{\partial z} d r+H_{m 1} .
$$

Through substituting Eq. (9) into Eq. (14),

$$
H_{g z}=H_{m 1}\left[1-\frac{\ln r / r_{0}}{\ln r_{1} / r_{0}}\left(1+\frac{S_{1}}{S_{2}}\right)\right]+\frac{\ln \left(r / r_{0}\right)}{\ln \left(r_{1} / r_{0}\right)} \frac{N_{1} I_{1}}{l_{k 1}} .
$$

As $H_{m 1} \ll N_{1} I_{1} / l_{k 1}$,

$$
H_{g z} \approx \frac{\ln r / r_{0}}{\ln r_{1} / r_{0}} \frac{N I_{1}}{l_{k 1}} .
$$

Define $\Phi_{e}$ as the average magnetic flux through coil

$$
\Phi_{e}=N \int_{\Omega} \mu H_{z} d V / l_{k},
$$

where $H_{z}$ is the axial magnetic field through the coil, $N$ represents the turn number of the corresponding coil (turn number of primary winding and secondary winding are $N_{1}$ and $N_{2}$, respectively). $\Omega$ and $l_{k}$ stand for volume and length of the coil, respectively. So mutual inductance $M$ is derived as

$$
M I_{1}=\Phi_{e 12}=N_{2} \int_{V_{k 2}} \mu H_{z} d V / l_{k 2} .
$$

Magnetizing inductance is derived as $L_{\mu}=M N_{1} / N_{2}$.

Assuming $l_{k 1}=l_{k 2}=l_{k}$, the leakage inductance $L_{s 1}$ of the primary coil is derived by

$$
L_{s 1} I_{1}=\Phi_{e s}=N_{1} \int_{V_{k 12}} \mu H_{z} d V / l_{k} .
$$

Here, $V_{k 2}$ stands for the area inside the secondary winding, and $V_{k 12}$ is the area between primary winding and the secondary winding. The area $V_{k 2}$ and $V_{k 12}$ is shown in Fig. 2. Assume leakage inductance $\left(L_{s 1}\right)$ of primary winding equals that $\left(L_{s 2}\right)$ of secondary winding, namely $L_{s 1}=L_{s 2}=L_{s}$. So inductance of the primary winding $L_{1}$, inductance of the secondary winding $L_{2}$, and the coupling coefficient of the transformer $k$ are calculated as

$$
\begin{aligned}
L_{1} & =L_{\mu}+L_{s}, \\
L_{2} & =\left(N_{2} / N_{1}\right)^{2} L_{1}, \\
k & =L_{u} / L_{1}=1-L_{s} / L_{\mu} .
\end{aligned}
$$

For case 1, 


$$
L_{\mu}=\frac{\mu_{0} \mu_{r} S_{1} S_{2} N_{1}^{2}}{\left(S_{1}+S_{2}\right) l_{k}}\left\{1-\frac{2 \cosh \left[\omega\left(l_{T}-l_{k}\right) / 2\right]}{\omega l_{k} \cosh \left(\omega l_{T} / 2\right)} \sinh \left(\frac{\omega l_{k}}{2}\right)\right\}+\frac{2 \pi \mu_{0} N_{1}^{2}}{l_{k}}\left[\frac{r_{1}^{3}}{6\left(r_{1}-r_{0}\right)}-\frac{5 r_{1}^{2}-4 r_{0}^{2}+5 r_{0} r_{1}}{36 \ln \left(r_{1} / r_{0}\right)}\right]
$$

and

$$
L_{s}=\frac{2 \pi \mu_{0} N_{1}^{2}}{l_{k}}\left[\frac{2 r_{1}^{3}-3 r_{0} r_{1}^{2}}{6\left(r_{1}-r_{0}\right)}-\frac{4 r_{1}^{2}-5 r_{0}^{2}-5 r_{0} r_{1}}{36 \ln \left(r_{1} / r_{0}\right)}\right]
$$

If $\omega l_{T} \ll 1, l_{T} \gg r_{1}$, Eq. (21) can be simplified as

$$
L_{\mu}=\frac{\pi \mu_{0} N_{1}^{2}\left(l_{T}-2 / 3 l_{k}\right)}{2 \ln \left(r_{1} / r_{0}\right)} .
$$

According to Eq. (20), when $l_{k} / l_{T}=0.6$, it is easy to find that the coupling coefficient $k$ of the transformer becomes largest if the length of the magnetic core $l_{T}$ is fixed. That is different from the result in Ref. [11] where the coupling coefficient becomes largest when $l_{k} / l_{T}=0.5$.

For case 2,

$$
\begin{aligned}
L_{\mu}= & \frac{\mu_{0} \mu_{r} S_{1} S_{2} N_{1}^{2} I_{1}}{\left(S_{1}+S_{2}\right) l_{k}}\left\{1-\frac{2\left\{\sqrt{\mu_{r}} \cosh \left[\omega\left(l_{T}-l_{k}\right) / 2\right]+\sinh \left[\omega\left(l_{T}-l_{k}\right) / 2\right]\right\}}{\omega l_{k}\left[\sqrt{\mu_{r}} \cosh \left(\omega l_{T} / 2\right)+\sinh \left(\omega l_{T} / 2\right)\right]} \sinh \left(\frac{\omega l_{k}}{2}\right)\right\} \\
& +\frac{2 \pi \mu_{0} N_{1}^{2}}{l_{k}}\left[\frac{r_{1}^{3}}{6\left(r_{1}-r_{0}\right)}-\frac{5 r_{1}^{2}-4 r_{0}^{2}+5 r_{0} r_{1}}{36 \ln \left(r_{1} / r_{0}\right)}\right],
\end{aligned}
$$

and the expression of $L_{s}$ is the same as Eq. (22).

\section{Effect of nonuniform distribution of current in primary coil}

Actually, the current distribution in primary winding is not as uniform as that in the magnetostatic-field condition. Because of skin effect, current density at the edge of the winding is larger than that at the middle at high frequency. Assuming current density distribution in primary winding is $j(z)$, Eq. (4) changes to the equation below:

$$
\left(1+S_{1} / S_{2}\right) H_{m 1}-\frac{\mu_{r} S_{1} \ln \left(r_{1} / r_{0}\right)}{2 \pi} \frac{\partial^{2} H_{m 1}}{\partial z^{2}}= \begin{cases}j(z) & 0<z<l_{k} / 2 \\ 0 & l_{k} / 2<z<l_{T} / 2 .\end{cases}
$$

Analyzing case 1 only, we get

$$
H_{m 1}(z)=\left\{-\frac{\omega \cosh \left[\omega\left(l_{T}-l_{k}\right) / 2\right] I\left(l_{k} / 2\right)+\sinh \left[\omega\left(l_{T}-l_{k}\right) / 2\right] I^{\prime}\left(l_{k} / 2\right)}{\omega \cosh \left(\omega l_{T} / 2\right)} \cosh (\omega z)\right\}+I(z), \quad 0<z<l_{k} / 2,
$$

and

$$
H_{g z} \approx \frac{\ln r / r_{0}}{\ln r_{1} / r_{0}} j(z)
$$

Then

$$
\begin{aligned}
L_{1}= & \frac{2 \mu S_{1} N_{1}}{l_{k} I_{1}}\left\{-\frac{\omega \cosh \left[\omega\left(l_{T}-l_{k}\right) / 2\right] I\left(l_{k} / 2\right)+\sinh \left[\omega\left(l_{T}-l_{k}\right) / 2\right] I^{\prime}\left(l_{k} / 2\right)}{\omega^{2} \cosh \left(\omega l_{T} / 2\right)} \sinh \left(\frac{\omega l_{k}}{2}\right)\right. \\
& \left.+\int_{0}^{l_{k} / 2} I(z) d z\right\}+\frac{2 \pi \mu_{0} N_{1}^{2}}{l_{k}}\left[\frac{r_{1}^{2}}{2}-\frac{r_{1}^{2}-r_{0}^{2}}{4 \ln \left(r_{1} / r_{0}\right)}\right],
\end{aligned}
$$

where $I(z)$ is the special solution to Eq. (25). We can approximately describe $j(z)$ by

$$
j(z)=\frac{N_{1} I_{1} b \cosh (b z)}{2 \sinh \left(b l_{k} / 2\right)},
$$

where $b$ describes the nonuniformity of current on primary winding. As $b$ becomes larger, current will focus on the edge of the primary winding gradually. At that point, the special solution to Eq. (25) is as

$$
I(z)=\frac{S_{2} \omega^{2} j(z)}{\left(\omega^{2}-b^{2}\right)\left(S_{1}+S_{2}\right)}
$$


We just consider an extreme situation where $b$ becomes infinitely large. In this case, the skin effect is so serious that current fully focuses on the edge of primary winding. Inductance of the primary winding is calculated as

$$
L_{1}=\frac{\mu_{0} \mu_{r} S_{1} S_{2} N_{1}^{2}}{\left(S_{1}+S_{2}\right) l_{k}}\left\{\frac{\sinh \left[\omega\left(l_{T}-l_{k}\right) / 2\right) \sinh \left(\omega l_{k} / 2\right)}{\cosh \left(\omega l_{T} / 2\right)}\right\}+\frac{2 \pi \mu_{0} N_{1}^{2}}{l_{k}}\left[\frac{r_{1}^{2}}{2}-\frac{r_{1}^{2}-r_{0}^{2}}{4 \ln \left(r_{1} / r_{0}\right)}\right] .
$$

If $\omega l_{T} \ll 1, l_{T} \gg r_{1}$, Eq. (31) can be simplified as Eq. (32), which is the same as the equation in Ref. [11]:

$$
L_{\mu}=\frac{\pi \mu_{0} N_{1}^{2}\left(l_{T}-l_{k}\right)}{2 \ln \left(r_{1} / r_{0}\right)} .
$$

Inductance of primary winding in case 2 can be obtained similarly when $b$ becomes infinitely large:

$$
L_{1}=\frac{\mu_{0} \mu_{r} S_{1} S_{2} N_{1}^{2}}{\left(S_{1}+S_{2}\right) l_{k}} \frac{\left\{\sqrt{\mu_{r}} \sinh \left[\omega\left(l_{T}-l_{k}\right) / 2\right]+\cosh \left[\omega\left(l_{T}-l_{k}\right) / 2\right]\right\}}{\left[\sqrt{\mu_{r}} \cosh \left(\omega l_{T} / 2\right)+\sinh \left(\omega l_{T} / 2\right)\right]} \sinh \left(\frac{\omega l_{k}}{2}\right)+\frac{2 \pi \mu_{0} N_{1}^{2}}{l_{k}}\left[\frac{r_{1}^{2}}{2}-\frac{r_{1}^{2}-r_{0}^{2}}{4 \ln \left(r_{1} / r_{0}\right)}\right] .
$$

Comparing Eq. (32) with Eq. (23), we can find that magnetizing inductance is largely decreased when the current distribution in the primary coil is seriously nonuniform. At the same time, according to Eqs. (26) and (27), when currents converge at the edge of primary winding, magnetic fields in the spacing between magnetic cores will also converge at the edge. According to electromagnetic induction law, the induced voltage on fringe turns of the secondary winding is larger than that of others because the magnetic flux getting through fringe turns is larger. It can lead to an overvoltage phenomenon at two ends of secondary winding when the transformer operates, especially at the larger end of secondary winding.

\section{DEVELOPMENT OF PULSE TRANSFORMER WITH OPEN MAGNETIC CORE}

\section{A. Development of pulse transformer}

To verify the correctness of the formulas derived in Sec. II, a pulse transformer with open magnetic core is designed. The photograph of the pulse transformer is shown in Fig. 3, and the diagram of the cross section is shown in Fig. 4. The transformer has a small size, whose dimensions are $250 \mathrm{~mm} \times 150 \mathrm{~mm}$ in length and diameter,

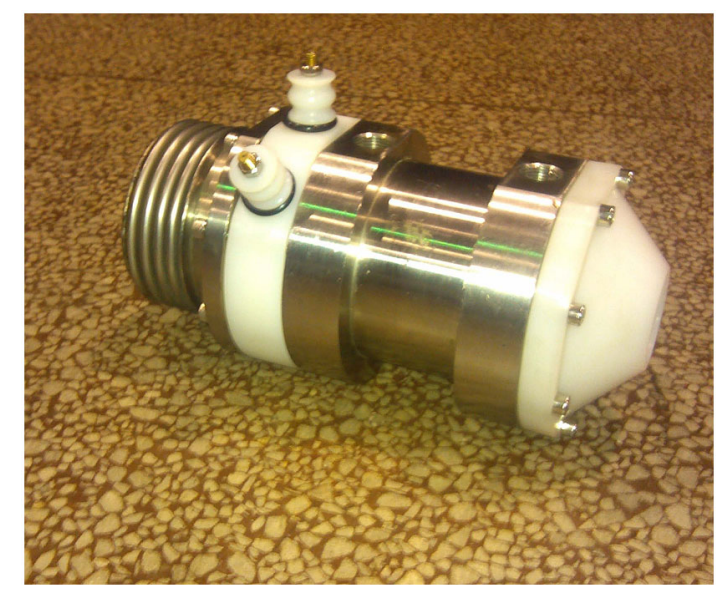

FIG. 3. Photograph of the pulse transformer. respectively. Structure parameters of the developed pulse transformer are shown as below: $u_{r}=1000, r_{0}=25 \mathrm{~mm}$, $r_{1}=41 \mathrm{~mm}, \quad S_{1}=1500 \mathrm{~mm}^{2}, \quad S_{2}=1100 \mathrm{~mm}^{2}, \quad l_{T}=$ $100 \mathrm{~mm}, l_{k 1}=l_{k 2}=60 \mathrm{~mm}, N_{1}=2.1, N_{2}=310$.

\section{B. Parameters test}

LCR meter Hp4284A is used to measure inductances of the transformer. Table I compares results of a variety of calculated and experimental values of pulse transformer inductances and coupling coefficients. Results are shown for two different cases: case 1, when the ends of the magnetic cores are buried in metal, and case 2, when the ends of the cores are open. The first three rows in Table I show results for magnetostatic calculations, finite elemental magnetostatic-field simulations, Eqs. (20)-(22) and (24) derived in this paper and Korovin's formula from Ref. [11]. Models for the finite elemental magnetostaticfield simulations are shown in Figs. 5 and 6, and PEC in the figures stands for perfect conductor. We expect the numeric error in these simulations to be very small because a small grid size was used in the calculations. The next three rows in Table I show limited experimental results at $100 \mathrm{~Hz}$ and then full $L_{1}, L_{2}$, and coupling coefficient $(k)$ results at

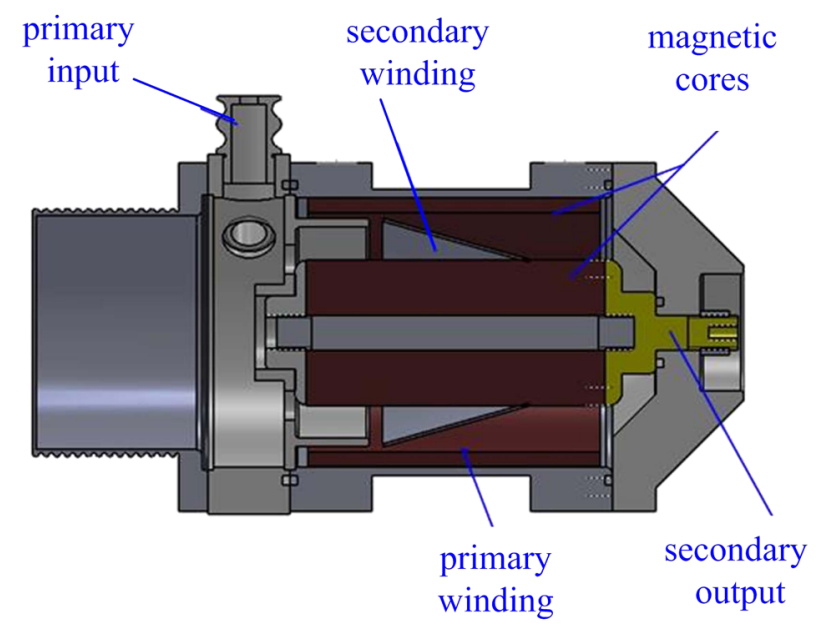

FIG. 4. Diagram of the cross section of the pulse transformer. 
TABLE I. Results of inductance parameters of the pulse transformer with four methods.

\begin{tabular}{|c|c|c|c|c|c|c|c|}
\hline Case 1 & $L_{1}$ & $L_{2}$ & $k$ & Case 2 & $L_{1}$ & $L_{2}$ & $k$ \\
\hline $\begin{array}{l}\text { Magnetostatic-field } \\
\text { simulations }\end{array}$ & $1.20 \mu \mathrm{H}$ & $26.50 \mathrm{mH}$ & 0.923 & $\begin{array}{l}\text { Magnetostatic-field } \\
\text { simulations }\end{array}$ & $1.37 \mu \mathrm{H}$ & $31.11 \mathrm{mH}$ & 0.935 \\
\hline Eqs. (19)-(21) & $1.26 \mu \mathrm{H}$ & $27.10 \mathrm{mH}$ & 0.910 & Eqs. (19), (21), and (23) & $1.46 \mu \mathrm{H}$ & $31.70 \mathrm{mH}$ & 0.918 \\
\hline Formula in [11] & $0.86 \mu \mathrm{H}$ & $18.90 \mathrm{mH}$ & 0.881 & Formula in [11] & $0.86 \mu \mathrm{H}$ & $18.90 \mathrm{mH}$ & 0.881 \\
\hline $100 \mathrm{~Hz}$ & & $31.05 \mathrm{mH}$ & & $100 \mathrm{~Hz}$ & & $31.89 \mathrm{mH}$ & \\
\hline $1 \mathrm{kHz}$ & $1.36 \mu \mathrm{H}$ & $31.05 \mathrm{mH}$ & 0.914 & $1 \mathrm{kHz}$ & $1.42 \mu \mathrm{H}$ & $29.28 \mathrm{mH}$ & 0.925 \\
\hline $10 \mathrm{kHz}$ & $1.10 \mu \mathrm{H}$ & $21.85 \mathrm{mH}$ & 0.905 & $10 \mathrm{kHz}$ & $1.24 \mu \mathrm{H}$ & $24.77 \mathrm{mH}$ & 0.915 \\
\hline Eq. (31) & $0.92 \mu \mathrm{H}$ & $20.00 \mathrm{mH}$ & & Eq. (33) & $1.13 \mu \mathrm{H}$ & $24.50 \mathrm{mH}$ & \\
\hline
\end{tabular}

frequencies of 1 and $10 \mathrm{kHz}$. Finally, the last row of Table I compares the experimental results at $10 \mathrm{kHz}$ with the results of calculations based on Eqs. (31) and (33) of this paper that abandon the magnetostatic restriction and allow for nonuniform current density in the coil.

The magnetostatic-field simulation values of $L_{1}$ and $L_{2}$ differ from the values obtained from Eqs. (20)-(24) in this work by only about $5 \%$ while the $k$ values differ by less than $2 \%$. When Korovin's equation for large aspect-ratio transformers is applied to our small aspect-ratio transformers in case 2 , it gives magnetizing inductance values that are about $30 \%-40 \%$ too low. The measured result for $L_{2}$ at $100 \mathrm{~Hz}$ is close to magnetostatic calculations for case 2

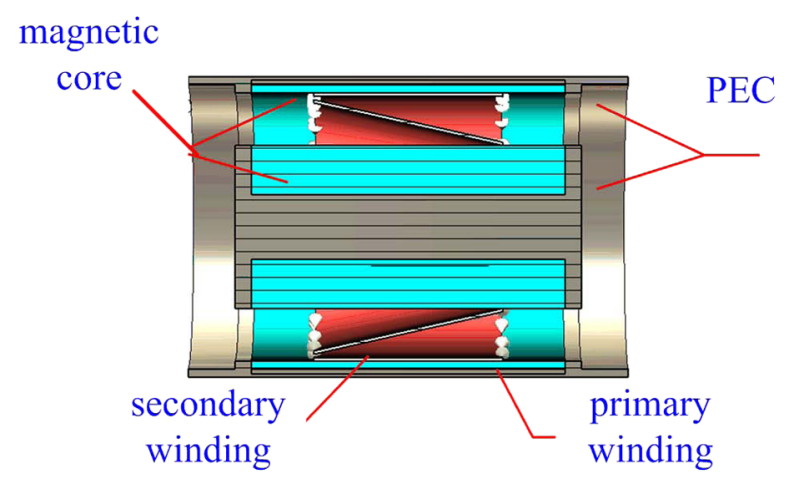

FIG. 5. Model in the finite elemental magnetostatic-field simulations for case 1 .

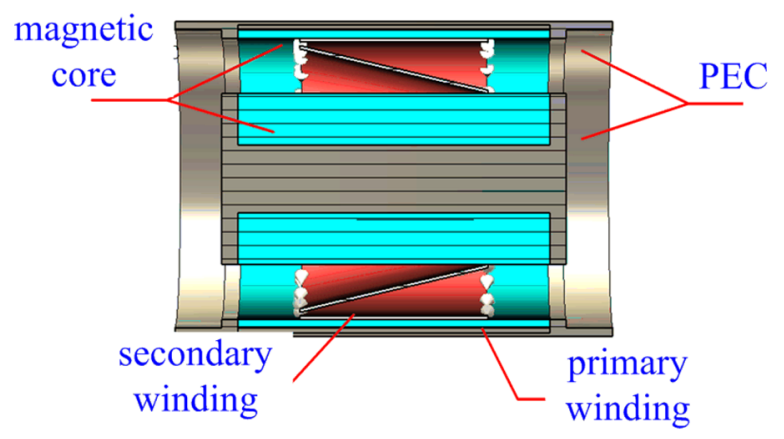

FIG. 6. Model in the finite elemental magnetostatic-field simulations for case 2 . with open core ends. For case 1, the low-frequency magnetic fields can penetrate the metal ends of the magnetic core and, hence, the magnetostatic results are not accurate.

As the test frequency increases from 100 to $10 \mathrm{kHz}$, the inductance of the transformer decreases sharply, becoming close to the nonmagnetostatic results obtained from Eqs. (31) and (33). These changes in inductance result from nonuniform current distribution in the primary winding, which reduces the magnetizing inductance and coupling coefficient of the transformer. So, it is meaningful to try to find a method to improve the current distribution in the primary winding.

\section{EXPERIMENTAL RESULTS OF PULSE TRANSFORMER AND ITS APPLICATION}

The test experiment on the transformer shown in Figs. 3 and 4 was carried out and schematics of the experiment are shown in Fig. 7. Most ends of the magnetic cores are open except the end where a copper secondary output exists, so the transformer design in this part is similar to case 2. Test results shows that primary inductance of the transformer $L_{1}=1.2 \mu \mathrm{H}$, secondary inductance $L_{2}=24 \mathrm{mH}$, and coupling coefficient $k=0.91$, which confirms to the calculated result from Eq. (33) in Table I. In Fig. 7, the pulse transformer is used to charge a $52 \mathrm{pF}$ high-voltage load capacitor $\left(C_{2}\right) . C_{1}$ is the primary energy-storage capacitor with $5 \mu \mathrm{F}$ capacitance. $R_{1}$ and $L_{k 1}$ are stray resistance and inductance of primary circuit, and $R_{2}$ is stray resistance of

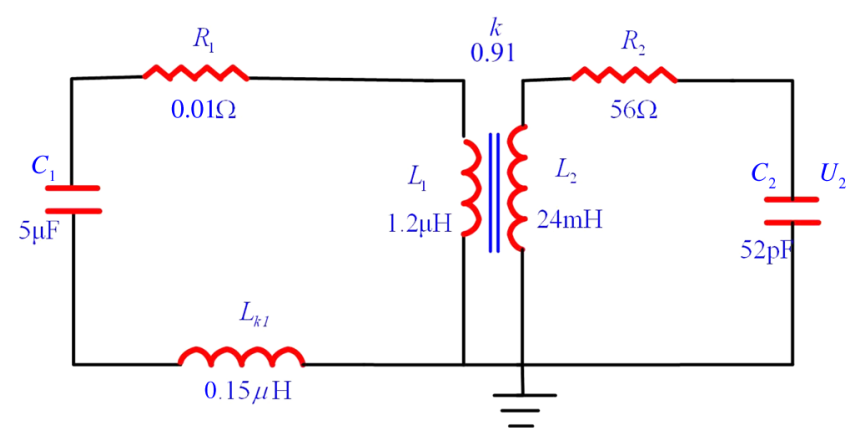

FIG. 7. Schematics of the test experiment on the pulse transformer. 


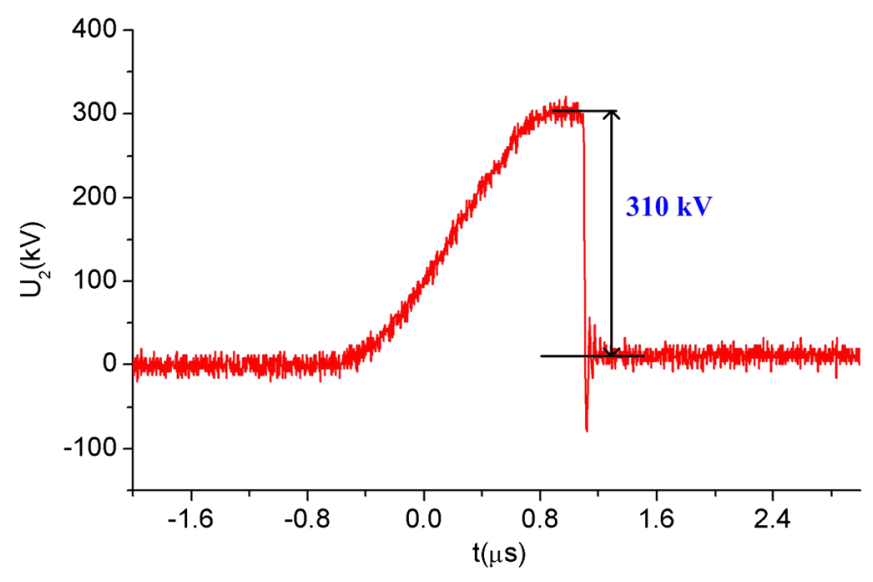

FIG. 8. Charging voltage waveform of load capacitor $C_{2}$.

the secondary circuit. The charging voltage waveform of $C_{2}$ is shown in Fig. 8. When initial charging voltage $U_{0}$ of $C_{1}$ is $1.9 \mathrm{kV}$, the peak charging voltage of $C_{2}$ is obtained as high as $310 \mathrm{kV}$ and the step-up ratio is $1: 163$. The peaking voltage time is $1.64 \mu \mathrm{s}$ [full width at half Maximum (FWHM) is $1 \mu \mathrm{s}]$.

However, as the work frequency of the transformer is as high as $300 \mathrm{kHz}$, the loss of magnetic cores has to be

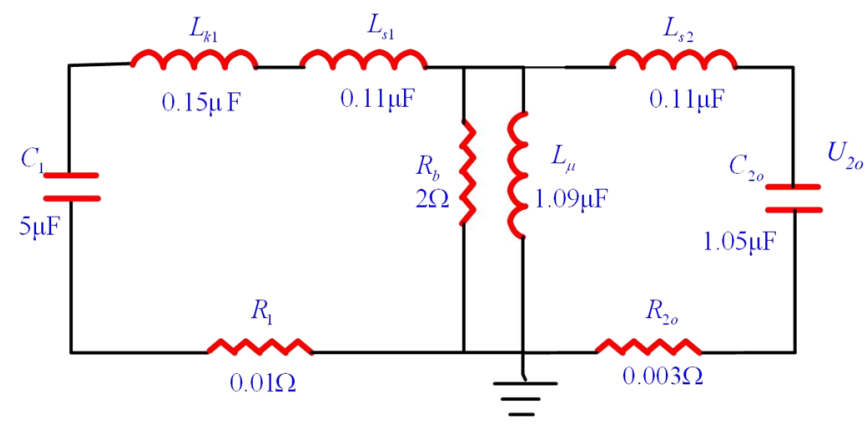

FIG. 9. Equivalent schematics used in the circuit simulation calculation.

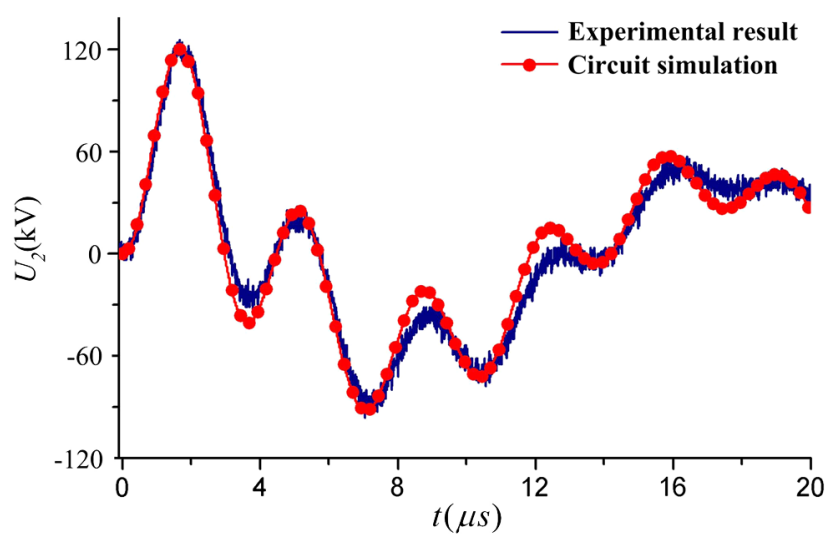

FIG. 10. Comparison of the simulation waveform and the experimental waveform. considered. According to Ref. [11], Fig. 9 shows the equivalent circuit of Fig. 7. $R_{2 o}, C_{2 o}$, and $U_{2 o}$ in Fig. 9 orderly correspond to $R_{2}, C_{2}$, and $U_{2}$ in Fig. 7, and $R_{2 o}=$ $\left(L_{1} / L_{2}\right) R_{2}, \quad C_{2 o}=\left(L_{1} / L_{2}\right) R_{1}, \quad U_{2 o}=\left(L_{1} / L_{2}\right)^{0.5} U_{2} . R_{b}$ ( $2 \Omega$ ) describes the loss in magnetic cores. Circuit simulation software is used to calculate the charging voltage of the secondary capacitor $C_{2}$, and the simulative output waveform of the schematics shown in Fig. 9 is presented in Fig. 10. The tested output voltage waveform when breakdown of the secondary switch does not occur is also presented in Fig. 10. (However $U_{2 o}$ has been transformed to $U_{2}$ in the figure.) Figure 10 shows that simulation waveform and the experimental waveform are superposed approximately, which demonstrates theoretical calculation of the inductance of the pulse transformer is correct.

Experimental results in Fig. 8 show that the pulse transformer can export voltage pulses of $310 \mathrm{kV}$, and its peaking charging time is $1.64 \mu \mathrm{s}$. Because the pulse transformer has advantages of small size, high step-up ratio, highvoltage outputs, wide response frequency band, and low costs, it can be used as a pulse source to charge the PFL in the lab.

\section{CONCLUSIONS}

Formulas for calculating inductances of the small aspect-ratio transformer are derived. The values of inductance of primary winding $L_{1}$ and inductance of secondary winding $L_{2}$ obtained from equations derived in this work differ from the magnetostatic-field simulation values by only about $5 \%$, while the coupling coefficient $(k)$ values differ by less than $2 \%$. Moreover, the effect of current nonuniformity of primary winding on magnetizing inductance is studied. This effect reduces magnetizing inductance and the coupling coefficient of the transformer, and can lead to an overvoltage phenomenon on the secondary winding. So it is meaningful to find a method to improve the current distribution in the primary winding. A small aspect-ratio pulse transformer with open magnetic core is developed. The transformer has a small size, whose dimensions are $250 \mathrm{~mm} \times 150 \mathrm{~mm}$ in length and diameter, respectively. Measured results of inductances of the transformer conform to the law obtained in this work, which demonstrates the theoretical calculation is correct. The tested experiment on the pulse transformer shows that the transformer can export high-voltage pulse with an amplitude of $310 \mathrm{kV}$ and full width at half maximum (FWHM) of $1 \mu \mathrm{s}$. Because the pulse transformer has advantages of small size, high step-up ratio, high-voltage outputs, wide response frequency band, and low costs, it can be used as a pulse source to charge the PFL in the lab.

[1] G. A. Mesyats, S. D. Korovin, A. V. Gunin, V. P. Gubanov, A. S. Stepchenko, D. M. Grishin, V.F. Landl, and P.I. Alekseenko, Laser Part. Beams 21, 197 (2003). 
[2] J. L. Liu, Y. Yin, B. Ge, T. W. Zhan, X. B. CHEN, J.H. Feng, T. Shu, J. D. Zhang, and X.X. Wang, Laser Part. Beams 25, 593 (2007).

[3] J.L. Liu, T. W. Zhan, J. Zhang, Z.X. Liu, J.H. Feng, T. Shu, J.D. Zhang, and X.X. Wang, Laser Part. Beams 25, 305 (2007).

[4] G. A. Mesyats, S. D. Korovin, V. V. Rostov, V. G. Sharp, and M. I. Yalandin, Proc. IEEE 92, 1166 (2004).

[5] G. A. Mesyats, S. N. Rukin, V. G. Shpak, and M. I. Yalandin, in Proceedings of the 4th Ultra-Wideband Short-Pulse Electromagnetics Conference, 1998 (IEEE, Tel-Aviv, 1998), pp. 1-9.

[6] J. Liu, X. Cheng, J. Pu, and X. Wang, IEEE Electr. Insul. Mag. 23, 20 (2007).

[7] J. C. Peng, G. Z. Liu, X. X. Song, and J. C. Su, Laser Part. Beams 29, 55 (2011).
[8] G. J. Rohwein, R. N. Lawson, and M.S. Clark, in Proceedings of the 8th IEEE International Pulse Power Conference, 1991 (IEEE, San Diego, 1991), pp. 968-970.

[9] J. Zhang, J. Dickens, M. Giesselmann, J. Kim, E. Jsristiansen, J. Mankowski, D. Garcia, and M. Kristiansen, in Proceedings of the 12th IEEE International Pulsed Power Conference (IEEE, Piscataway, NJ, 1999), pp. 704-707.

[10] M. Istenic, B. M. Novac, J. Luo, R. Kumar, and I. R. Smith, IEEE Trans. Plasma Sci. 36, 2644 (2008).

[11] S. D. Korovin, Foreign Nucl. Test Technol. 19, 1 (1996) (in Chinese).

[12] X. B. Zhang and J. C. Su, High Power Laser Part. Beams 21, 943 (2009) (in Chinese). 